\title{
Article \\ Floquet spectral almost periodic modulation of massive finite and infinite strongly coupled arrays: Dense massive MIMO, intelligent surfaces, $5 \mathrm{G}$ and $6 \mathrm{G}$ applications
}

\author{
HAMDI Bilel ${ }^{1, *}$ (1) and AGUILI Taoufik ${ }^{2}$ \\ 1 Sys'Com Laboratory, National Engineering School of Tunis, University Tunis El Manar, Tunis, Tunisia; \\ hbilel.enit@gmail.com \\ 2 Sys'Com Laboratory, National Engineering School of Tunis, University Tunis El Manar, Tunis, Tunisia; \\ Taoufik.aguili@gmail.com \\ * Correspondence: hbilel.enit@gmail.com ; Tel.: +32 466422407
}

\begin{abstract}
In this paper, we introduce a new formulation based on Floquet (Fourier) spectral analysis combined with a spectral modulation technique (and its spatial form) to study strongly coupled sublattices predefined in the infinite and large finite extent of almost periodic antenna arrays (e.g metasurfaces). This analysis is very relevant for dense massive MIMO, intelligent surfaces, $5 G$, and $6 \mathrm{G}$ applications (used for very small areas with a large number of elements such as millimeter and terahertz waves applications). The numerical method that is adopted to model the structure is the method of moments simplified by equivalent circuits MoM GEC. Other numerical methods (as the ASM array scanning method and windowing Fourier method) used this analysis in their kernel that to treat periodic and pseudo-periodic (or quasi-periodic) arrays.
\end{abstract}

Keywords: Floquet analysis; MoM method ; Almost periodic antenna arrays; Fourier analysis; strong mutual coupling ; Dense massive MIMO; MM and THz waves ; $5 \mathrm{G}$ and 6G applications

\section{Introduction}

Antenna arrays, and in particular dense (or massive) coupled almost-periodic antenna arrays, have been of great interest in telecommunications and RF electronic applications (such as dense massive MIMO applications, smart surfaces, 5G and 6G) [11] -[14], including those used for very small surfaces with large numbers of elements such as millimeter and terahertz array applications. Therefore, the spectrum analysis based on a Fourier transformation (in the Floquet domain) is proposed to simplify the EM calculation on an elementary cell surrounded by periodic walls, as explained in [9]-[15] (in other research, they use periodic Green's functions) [17]-[28]. In the bibliography and recent studies, only spatial modulation techniques have been proposed to study periodic systems with large sizes [33]-[36]. Except in our case, a Fourier spectral analysis is presented to introduce a spectral modulation technique and its spatial equivalent (Fourier and Fourier inverse) to study strongly coupled sub arrays in an infinite and large finite almost periodic support [6],[30]-[32] . In this context, several numerical methods such as FDTD and FEM and other integral methods like the method of moments and full-wave methods[16] are proposed to resolve the given problem. In our work, we are interested only in the method of moments combined with equivalent circuits and Floquet analysis to study the suggested structure with the principle of modulation. This work is divided into four parts: we start with an explication of the almost-periodic modal (or spectral) modulation and its spatial equivalent to examining strongly coupled cells [1]-[5]. Then, we applied MoM-GEC as a numerical method to solve the proposed problem [7]-[15], [29] . Next, several numerical results are presented to confirm the validity of the approach. Finally, some conclusions are established. 


\section{Almost periodic spectral (Floquet) modulation and its spatial equivalent to study strongly coupled arrays}

The concept is a signal processing concept for a filter with a periodic spectral response, as shown in [2]-[4]. His response is described as an impulse response function that is given by:

$$
\int_{-\infty}^{\infty} K(\Omega-\alpha) u(\alpha) d \alpha=V(\Omega)
$$

Its Fourier representation of equation (1) (in the spatial domain) yields to

$$
H(x) U(x)=V(x)
$$

From the transformations by way of analogy, that we take into account, we note that $x$ is a spatial coordinate and $\alpha \in\left[-\frac{\pi}{d}, \frac{\pi}{d}\right]$ is a spectral coordinate in the Brillouin domain. Note that also $\Omega$ is a spectral coordinate, as $\alpha$. where $H(x)$ is the Fourier transform of $K(\Omega)$ and is defined as the optical (or optoelectronic [2] , [3] ) transfer function, $\mathrm{U}(\mathrm{x})$ and $\mathrm{V}(\mathrm{x})$ are the Fourier transforms of $u(\alpha)$ and $V(\Omega)$, respectively. More details are provided in [2].

\subsection{Modulation in infinite Almost periodic arrays}

Let us consider $f_{\alpha}(x)$ a spectral periodic response for infinite array that is written [5], [14] :

$$
f_{\alpha}(x)=\sum_{n=-N}^{N=+\infty} f_{n}(x) e^{+j n \alpha d_{x}}
$$

$\alpha$ is a continuous Floquet modes $\alpha \in\left[-\frac{\pi}{d}, \frac{\pi}{d}\right]$ and $\mathrm{x}$ ( or $x_{n}=n d_{x}$ ) represent the position in the spatial domain (a spatial distribution). with

$$
f_{n}(x)=\frac{d}{2 \pi} \int_{-\frac{\pi}{d}}^{\frac{\pi}{d}} f_{\alpha}(x) e^{-j \alpha n d_{x}} d \alpha
$$

and $f_{n}(x)=f_{0}(x-n d), n \in \mathbb{Z} \cdot f_{n}(x)$ is a periodic function.

Now, let's put the given spectral modulation [2]:

$$
U_{m o d}^{\infty}(\alpha)=u(\alpha) f_{\alpha}(x)=u(\alpha) \sum_{n=-N}^{N=+\infty} f_{n}(x) e^{+j \alpha n d_{x}}
$$

Next, we are considering : $T F\left(U_{\text {mod }}^{\infty}(\alpha)\right)=U_{\text {mod }}^{\infty}(x)$

Possible to take $T F^{-1}\left(U_{\text {mod }}^{\infty}(\alpha)\right)=U_{\text {mod }}^{\infty}(x)($ it depends the Fourier notation) hence,

$$
U_{\text {mod }}^{\infty}(x)=\sum_{n=-N}^{N=+\infty} f_{n} U(x-n d)
$$

A simple demonstration from (5) to (6) is provided :

$$
\begin{array}{r}
U_{\text {mod }}^{\infty}(x)=T F^{-1}\left(U_{\text {mod }}^{\infty}(\alpha)\right) \\
=T F^{-1}\left(u(\alpha) \sum_{n=-N}^{N=+\infty} f_{n} e^{+j \alpha x}\right) \\
=\sum_{n=-N}^{N=+\infty} f_{n} T F^{-1}\left(u(\alpha) e^{+j \alpha x}\right) \\
=\sum_{n=-N}^{N=+\infty} f_{n}\left(\frac{d}{2 \pi} \int_{-\frac{\pi}{d}}^{\frac{\pi}{d}} u(\alpha) e^{+j \alpha x} e^{-j \alpha n d} d \alpha\right)
\end{array}
$$




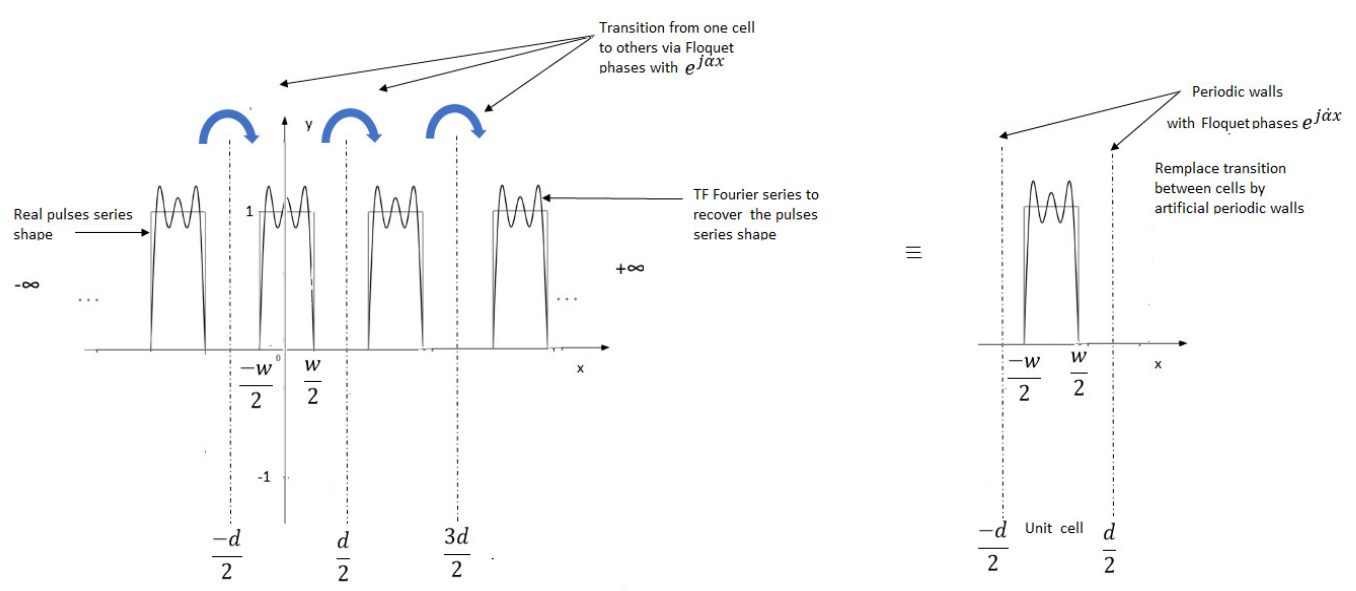

Figure 1. Construction of $f_{\alpha}(x)$ as given in equations (9), (10) and (11)

$$
\begin{array}{r}
=\sum_{n=-N}^{N=+\infty} f_{n}\left(\frac{d}{2 \pi} \int_{-\frac{\pi}{d}}^{\frac{\pi}{d}} u(\alpha) e^{+j \alpha(x-n d)} d \alpha\right) \\
=\sum_{n=-N}^{N=+\infty} f_{n} U(x-n d)
\end{array}
$$

The equation (7) is established refering to the theorem of aliasing given in [41] and the equation (6.107) of [42].

Finally, a spatial modulation is derived from the equation (2) [2] , [6]

$$
V_{\text {mod }}^{\infty}(x)=H(x) U_{m o d}^{\infty}(x)=H(x) \sum_{n=-N}^{N=+\infty} f_{n} U(x-n d)
$$

Notice that my old published work [7] on rectangular pulse functions can be a special case of this process of analysis and development. Where $f_{n, \alpha}=\frac{W}{2 d} \operatorname{sinc}\left(\left(\frac{2 n \pi}{d}+\alpha\right) \frac{w}{2}\right)$ are the Fourier series coefficients of the periodic pulse train (in the presence of Floquet modes), as described in [39].

For more details, following the pulse (or impulse ) trains, we can introduce the Floquet phases as follows [5], [14] :

$$
\begin{aligned}
& f_{\alpha}(x)= \sum_{n=-N}^{N=+\infty} \operatorname{rect}(x-n d) e^{+j \alpha x} \\
&=\sum_{n=-N}^{N=+\infty} \operatorname{rect}_{n}(x) e^{+j \alpha x}
\end{aligned}
$$

Note that : $\sum_{n=-N}^{N=+\infty} \operatorname{rect}_{n}(x)=\sum_{n=-N}^{N=+\infty} f_{n} e^{+j \frac{2 n \pi}{d} x}$ (for a standard rectangular pulse trains), which explains how to recover (reconstruct) pulses train by means the Fourier series, as shown in figure(1) and explained in [39] and [40] (see subsection periodic pulse and impulse trains in [39] ). By adding the Floquet contribution $e^{+j \alpha x}$, we obtain the definition $f_{\alpha}(x)=\sum_{n=-N}^{N=+\infty} \operatorname{rect}(x-n d) e^{+j \alpha x}$ (See figure(1)).

Rect is a rectangular function with a width $\mathrm{W}$. 
Then, we can develop a series of rectangle functions into a series of Fourier functions, which allows us to write : (See to figure (1) and [7] )

$$
f_{\alpha}(x)=\sum_{n=-N}^{N=+\infty} f_{n, \alpha} e^{+j \frac{2 n \pi}{d}} e^{+j \alpha x}=\sum_{n=-N}^{N=+\infty} f_{n, \alpha} e^{+j\left(\frac{2 n \pi}{d}+\alpha\right) x}
$$

we can note : $K_{x, n}=\frac{2 n \pi}{d}+\alpha$ as an wavenumbers leads to decompose

$$
f_{\alpha}(x)=\sum_{n=-N}^{N=+\infty} f_{n, \alpha} e^{+j K_{x, n} x}
$$

With

$$
\begin{array}{r}
f_{n, \alpha}=\frac{1}{d} \int_{-\frac{d}{2}}^{\frac{d}{2}} f_{\alpha}(x) e^{-j K_{x, n} x} d x \\
=\frac{1}{d} \int_{-\frac{w}{2}}^{\frac{w}{2}} e^{-j K_{x, n} x} d x \\
=\frac{W}{2 d} \operatorname{sinc}\left(K_{x, n} \frac{w}{2}\right)=\frac{W}{2 d} \operatorname{sinc}\left(\left(\frac{2 n \pi}{d}+\alpha\right) \frac{w}{2}\right)
\end{array}
$$

The equation (12) is proven based on the example (example 3.17) of [39].

Now let us apply the superposition theorem of equation (10) (based on Floquet states) to generate the spatial solution [18], [28]: it is also called modulation (see equation 7)

$$
\begin{array}{r}
\frac{d}{2 \pi} \int_{-\frac{\pi}{d}}^{\frac{\pi}{d}} f_{\alpha}(x) e^{-j \alpha x} d \alpha=T F^{-1}\left(f_{\alpha}(x)\right) \\
=\frac{d}{2 \pi} \int_{-\frac{\pi}{d}}^{\frac{\pi}{d}}\left(\sum_{n=-N}^{N=+\infty} f_{n, \alpha} e^{+j\left(\frac{2 n \pi}{d}+\alpha\right) x}\right) e^{-j \alpha x} d \alpha \\
=\frac{d}{2 \pi} \int_{-\frac{\pi}{d}}^{\frac{\pi}{d}}\left(\sum_{n=-N}^{N=+\infty} f_{n, \alpha} e^{+j\left(\frac{2 n \pi}{d}\right) x}\right) d \alpha \\
=\frac{d}{2 \pi} \int_{-\frac{\pi}{d} \sum_{n=-N}^{\frac{\pi}{d}} \operatorname{lect}_{n}(x)}^{N=+\infty} d \alpha \\
=\frac{d}{2 \pi} \int_{-\frac{\pi}{d}}^{\frac{\pi}{d}} \sum_{n=-N}^{N=+\infty} \operatorname{rect}(x-n d) d \alpha \\
=\frac{d}{2 \pi} \sum_{n=-N}^{N=+\infty} r e c t(x-n d) \int_{-\frac{\pi}{d}}^{\frac{\pi}{d}} d \alpha \\
=\frac{d}{2 \pi} \sum_{n=-N}^{N=+\infty} \operatorname{rect}(x-n d) \frac{2 \pi}{d} \\
=\sum_{n=-N}^{N=+\infty} \operatorname{rect}(x-n d)
\end{array}
$$

The spatial solution: is a periodic pulses series

$$
\text { is similar to }=U_{\text {mod }}^{\infty}(x) \text { when }(u(\alpha)=1)
$$

What we get in (13) is similar to equation (14) of my published work [7] (and the equation (4) of WATANABE reference [6]). Also, it is of the same type as equation (6) and the expansion that follows in equation (7). Then, a spatial modulation that has been performed in equation (8) is being followed . 


\subsection{Modulation in finite Almost periodic arrays}

As previously explained in [10], [14], the interactions between cells in spectral domain for periodic finite array are governed by a discrete phase law such that $\alpha_{p}=$ $\frac{2 \pi p d}{D}=\frac{2 \pi p}{N}$ (with $-\frac{N_{x}}{2} \leq p \leq-\frac{N_{x}}{2}-1$ ) which comes from a rule of three math reasoning.

For a large period of finite array $D \longrightarrow 2 \pi$ ( $2 \pi$ is the hole interval of phases ).

For a local period of finite array $p d \longrightarrow \alpha_{p}=$ ? (is the spectral contribution for one cell in position $p d$ ) ( $\mathrm{p}$ is the index position in finite array and $\mathrm{d}$ is the local period ).

So, $\Longrightarrow \alpha_{p}=\frac{2 \pi p}{N}$, where $N_{x}$ is the total number of elements in finite array and $\mathrm{p}$ is the index position [22].

Figure (2) explains how to discretize the phases from the infinite case to the finite case.
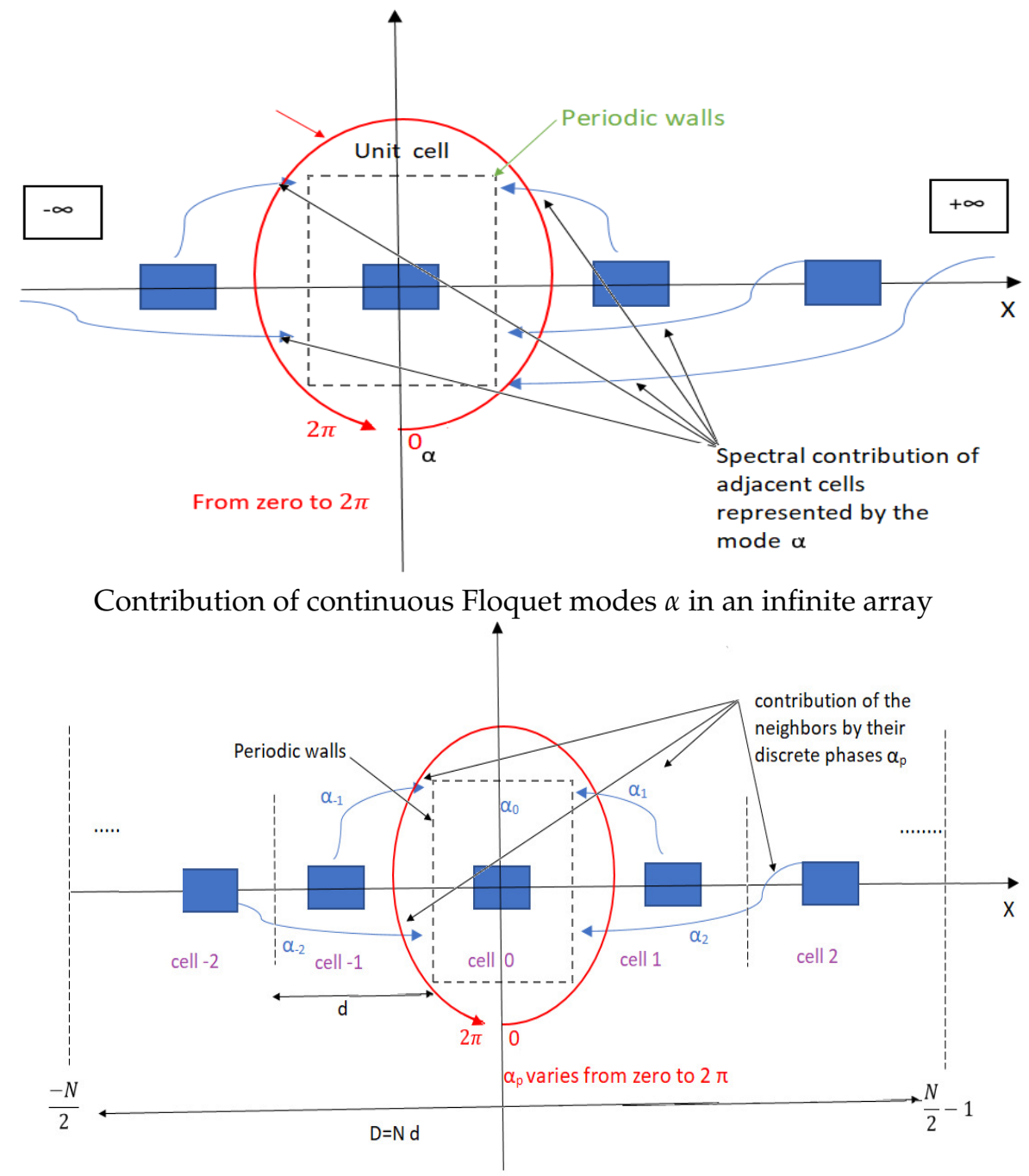

Contribution of discrete Floquet modes $\alpha_{p}$ in finite array

Figure 2. Spectral representation of the interactions of a unit cell with its neighbors (infinite and finite cases ) (valid for strong coupling interaction by using Floquet phases )

According to the same figure (2), each cell interacts spectrally with its neighbors through the continuous Floquet modes $\alpha$ (or the phase shift $e^{+j \alpha x}$ ) in infinite case and respectively 
$\alpha_{p}$ (or the phase shift $e^{+j \alpha_{p} x}$ ) in finite case.

which allows writing the spectral solution as the sum of discrete Floquet states, [5],[14],

$$
f_{\alpha_{p}}(x)=\frac{1}{\sqrt{N_{x}}} \sum_{n=-\frac{N_{x}}{2}}^{\frac{N_{x}}{2}-1} f_{n}(x) e^{+j \alpha_{p} n d_{x}}
$$

and

$$
f_{n}(x)=\frac{1}{\sqrt{N_{x}}} \sum_{p=-\frac{N_{x}}{2}}^{\frac{N_{x}}{2}-1} f_{\alpha_{p}}(x) e^{-j \alpha_{p} n d_{x}}
$$

In the same way $f_{n}(x)=f_{0}(x-n d)$ with $-\frac{N_{x}}{2} \leq n \leq-\frac{N_{x}}{2}-1$.

we can rewrite the spectral modulation law for a discrete Floquet modes,[2],

$$
\begin{array}{r}
U_{\text {mod }}^{\text {Finite }}\left(\alpha_{p}\right)=u\left(\alpha_{p}\right) f_{\alpha_{p}}(x) \\
=u\left(\alpha_{p}\right) \frac{1}{\sqrt{N_{x}}} \sum_{n=-\frac{N_{x}}{2}}^{\frac{N_{x}}{2}-1} f_{n}(x) e^{+j \alpha n d_{x}}
\end{array}
$$

Thus, the DFT is written as $\operatorname{DFT}\left(U_{\text {mod }}^{\text {Finite }}\left(\alpha_{p}\right)\right)=U_{\text {mod }}^{\text {Finite }}\left(x_{i}\right)$ with $-\frac{N_{x}}{2} \leq i \leq-\frac{N_{x}}{2}-1$ from which

$$
U_{\text {mod }}^{\text {Finite }}(x)=\frac{1}{\sqrt{N_{x}}} \sum_{n=-\frac{N_{x}}{2}}^{\frac{N_{x}}{2}-1} f_{n} U(x-n d)
$$

Eventually

$$
\begin{array}{r}
V_{\text {mod }}^{\text {Finite }}(x)=H(x) U_{\text {mod }}^{\text {Finite }}(x) \\
=H(x) \frac{1}{\sqrt{N_{x}}} \sum_{n=-\frac{N_{x}}{2}}^{\frac{N_{x}}{2}-1} f_{n} U(x-n d)
\end{array}
$$

\section{MoM-GeC modelization based on Floquet analysis}

The problem formulation is already explained in [9]-[15] .

\section{Numerical results}

A part of our results was presented in [7], [8], [10] and [14]. Let us now display the other obtained results.

This approach can be applied to FMCW radar antennas (to scan the radiation beam produced by a very small areas of antenna system), as well as to antennas that are massively placed in a coupled almost-periodic antenna array. The provided antenna example can be used to show how to model a $77 \mathrm{GHz}(2 \times 4)$ antenna array for frequency modulated continuous wave (FMCW) radar applications. The availability of antennas and antenna arrays in and on vehicles has become ordinary with the inclusion of remote crash recognition and aversion systems, as well as lane departure alerting systems. The two frequency bands appropriated for these systems are approximately $24 \mathrm{GHz}$ and 77 $\mathrm{GHz}$, respectively. In this model, we will consider the microstrip patch antenna as a phased array radiator. The dielectric substrate is air. According to figures (3) and (5), the patch antenna has its first resonance (parallel resonance) at 23.7 and $76 \mathrm{GHz}$. It is a common practice to shift this resonance to 24 and $77 \mathrm{GHz}$ by scaling the length of the planar dipole antenna, as described in [8].

The next stage is to reconfirm the reflection coefficient of the planar antenna dipole, as 
depicted in the figures (4) and Fig.1 of [7]. The purpose of this check is to consider a good impedance match. It is very common to highlight the value as a limit value for the calculation of the bandwidth of the antenna. The deepest minima at $24 \mathrm{GHz}$ and $77 \mathrm{GHz}$ indicated a good fit with $120 \pi$. The bandwidths of the antennas are roughly $1 \mathrm{GHz}$ and 2 GHz correspondingly. Thus, the spectrum bands are $23.5 \mathrm{GHz}$ to $25.5 \mathrm{GHz}$ and $76.5 \mathrm{GHz}$ to $77.5 \mathrm{GHz}$. Finally, in terms of input impedances and $S$ parameters, a good comparison between the adopted MoM GeC method and the reference [37] is obtained .

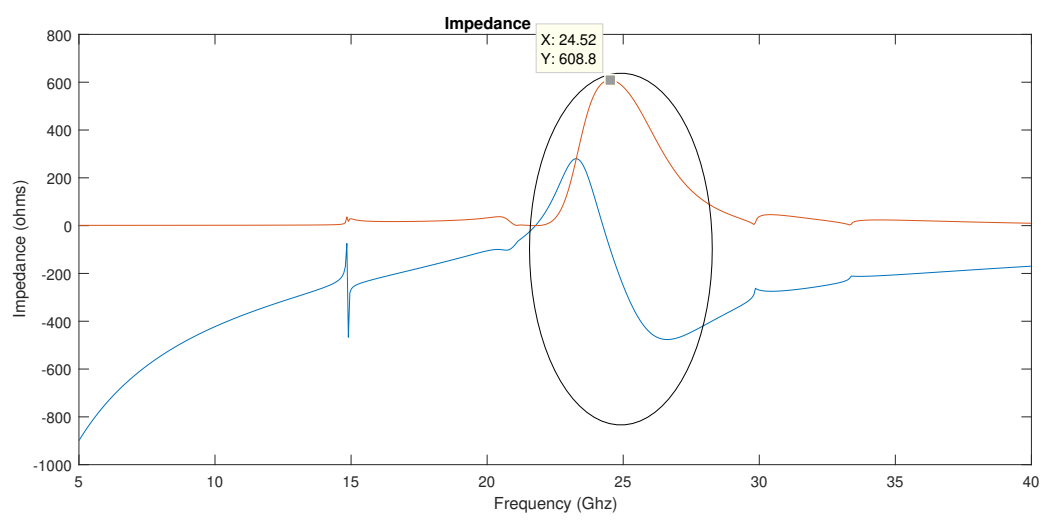

Figure 3. Impedance variation against frequency band around $24 \mathrm{GHz}$ : obtained by the $\mathrm{MoM} \mathrm{GeC}$ method

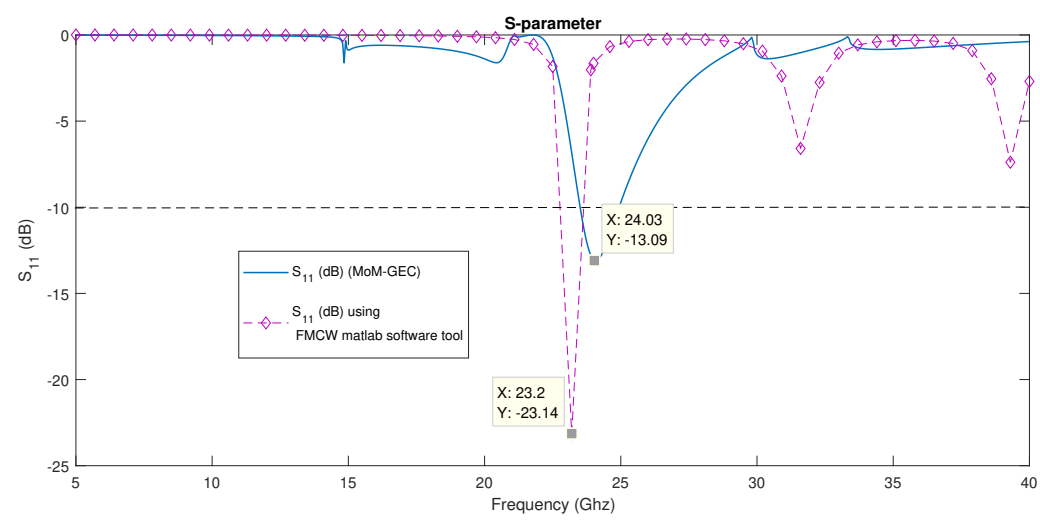

Figure 4. $S_{11}$-parameter $(\mathrm{dB})$ variation against frequency band around $24 \mathrm{GHz}$ : a comparison between the MoM GeC and a matlab tool (see reference [37] )

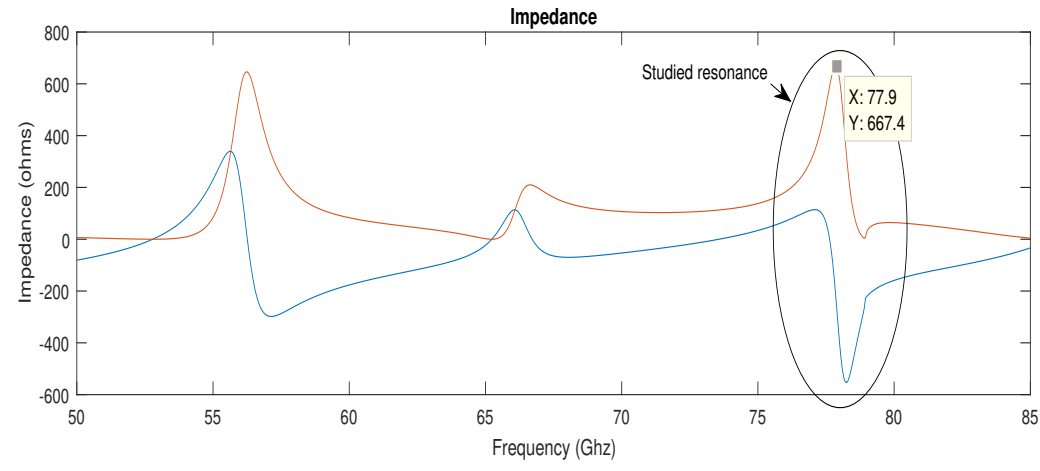

Figure 5. Impedance variation against frequency band around $77 \mathrm{GHz}$ : obtained by the MoM GeC method 


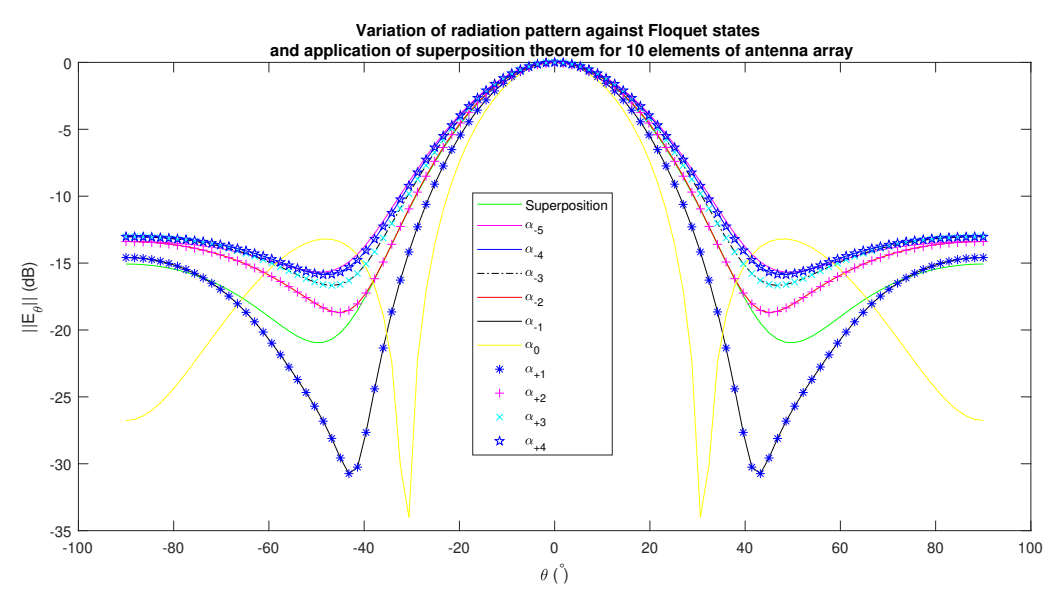

Figure 6. Variation of radiation pattern against Floquet states and application of superposition theorem for 10 elements of antenna array (uni-dimentionnal configuration) at $77 \mathrm{GHz}$ : obtained by the MoM GeC method

Table 1: Directivity versus some Floquet states (considering 100 antenna arrays) and the superposition theorem (or the modulation as explained in formula (13) which transformed to study a finite array ) for $\phi_{s}=0, \theta_{s}=30^{\circ}$ steering angles (used for 5G application)

\begin{tabular}{lc}
\hline Floquet states & Directivity values $(\mathrm{dB})$ at 24 Ghz \\
\hline$\left(\alpha_{-49}, \beta=0\right)$ & 23.0200 \\
$\left(\alpha_{-10}, \beta=0\right)$ & 22.5953 \\
$\left(\alpha_{+20}, \beta=0\right)$ & 22.8874 \\
$\left(\alpha_{+30}, \beta=0\right)$ & 23.2055 \\
\hline Superposition & 23.3009 \\
\hline Floquet states & Directivity values $(\mathrm{dB})$ at 77 Ghz \\
\hline$\left(\alpha_{-49}, \beta=0\right)$ & 22.8771 \\
$\left(\alpha_{-10}, \beta=0\right)$ & 24.1083 \\
$\left(\alpha_{+20}, \beta=0\right)$ & 23.4323 \\
$\left(\alpha_{+30}, \beta=0\right)$ & 23.1353 \\
\hline Superposition & 23.5783 \\
\hline
\end{tabular}
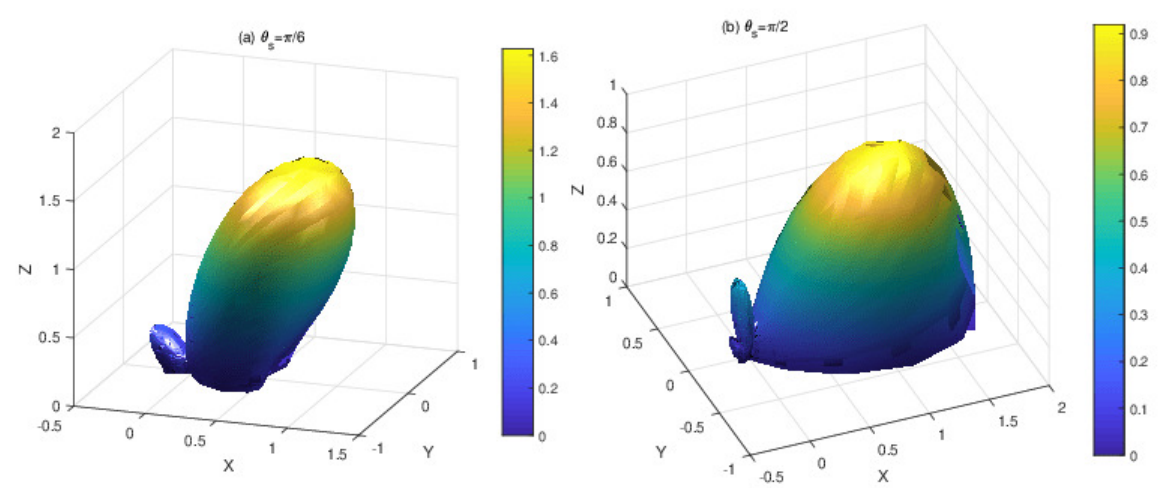

Figure 7. Variation of radiation pattern against Floquet states and application of superposition theorem for 100 elements of antenna array (uni-dimentionnal configuration) at $77 \mathrm{GHz}$ : obtained by the MoM GeC method

Therefore, the radiation pattern response for a small $2 \times 4$ antenna array is proposed based on the Floquet analysis (via the superposition theorem). Let us assume that the radar antenna system operates at $77 \mathrm{GHz}$ with a bandwidth of $700 \mathrm{MHz}$. The following 
Table 2: Directivity versus Floquet states and the superposition theorem (or the modulation as explained in formula (13) which transformed to study a finite array) for $\phi_{s}=0, \theta_{s}=45^{\circ}$ steering angles(FMCW radar application)

\begin{tabular}{lc}
\hline Floquet states & Directivity values $(\mathrm{dB})$ at $24 \mathrm{Ghz}$ \\
\hline$\left(\alpha_{-2}, \beta_{-1}\right)$ & 21.7249 \\
$\left(\alpha_{-1}, \beta_{-1}\right)$ & 26.7351 \\
$\left(\alpha_{0}, \beta_{-1}\right)$ & 21.1290 \\
$\left(\alpha_{+1} \beta_{-1}\right)$ & 20.8615 \\
$\left(\alpha_{-2}, \beta_{0}\right)$ & 20.6344 \\
$\left(\alpha_{-1}, \beta_{0}\right)$ & 21.0667 \\
$\left(\alpha_{0}, \beta_{0}\right)$ & 14.8990 \\
$\left(\alpha_{+1}, \beta_{0}\right)$ & 21.0737 \\
\hline Superposition & 21.0455 \\
\hline Floquet states & Directivity values $(\mathrm{dB})$ at $77 \mathrm{Ghz}$ \\
\hline$\left(\alpha_{-2}, \beta_{-1}\right)$ & 22.3762 \\
$\left(\alpha_{-1}, \beta_{-1}\right)$ & 18.0573 \\
$\left(\alpha_{0}, \beta_{-1}\right)$ & 21.9229 \\
$\left(\alpha_{+1} \beta_{-1}\right)$ & 22.1662 \\
$\left(\alpha_{-2}, \beta_{0}\right)$ & 20.9345 \\
$\left(\alpha_{-1}, \beta_{0}\right)$ & 21.3088 \\
$\left(\alpha_{0}, \beta_{0}\right)$ & 13.7990 \\
$\left(\alpha_{+1}, \beta_{0}\right)$ & 21.3088 \\
\hline Superposition & 21.7022 \\
\hline
\end{tabular}

figure Fig.1. of [8] shows the spatial radiation pattern of the resulting planar antenna using the superposition theorem of 2x4 Floquet radiation examples. Then, the sum of the discrete Floquet radiation patterns assigned to FMCW radar permits the prediction of the global spatial radiation pattern (what is called spatial modulation ). A good comparison of the given numerical radiation pattern and the radiation obtained by patch array and cosine array is presented in figure Fig.3. of [8]. After validating the FMCW radar, we propose to evaluate the approach for a very large number of elements that uses the same frequency band at 24 and $77 \mathrm{GHz}$ (for example a lattice of 100 elements ). Fig. 6 gives an example of the superposition theorem (or a spatial modulation) for a large array to generate a spatial radiation pattern through the addition of the radiation patterns of Floquet states. After that, Fig.4. of [8] presents the variation of the spatial radiation pattern (obtained using Floquet analysis) in the function of steering angles for 100 antenna elements that distributed in uni-dimensional configuration ( for 5G application). In the same way, Fig.7 and Fig.5. of [8] show the variation of 3D radiation pattern against different steering angles that are described with $\left(\theta_{0}=45, \phi_{0}=0\right)$ and $\left(\theta_{0}=90, \phi_{0}=0\right)$, and respectively in cartesian coordinates and $(\mathrm{u}, \mathrm{v})$ space. Following the same study, the tables (1) and (2) show the directivity values for each Floquet state and the superposition theorem (in the 2 cases of the FMCW radar and the 5G application), with 2 different steering angles and at the frequencies $24 \mathrm{GHz}$ and $77 \mathrm{GHz}$. From figures (Fig.1. of [8]) and (6) we can see that the radiation patterns are nearly identical and verify the condition where the directivities are similar for both the Floquet states and the superposition theorem. This is why, in both tables (1) and (2) we find that all directivity values are identical (for the Floquet states as well as for the superposition), even when we change the steering angle. Knowing that generally, the directivity value close to 20 $\mathrm{dB}$ satisfies a narrow beam angle of about 20 degrees, as shown graphically in figure ( 7.a).

\section{Conclusion}

In this paper, we have illustrated the principle of Floquet spectral modulation based on the Fourier analysis (and its spatial form ) to study almost periodic sub-arrays (with 
finite size) in the presence of strong mutual coupling interaction, defined on infinite support (or really largely finite size). Knowing that this study is very useful for the new generation of technologies based on millimeter and terahertz waves in phased arrays, for example in dense massive MIMO, smart surfaces, 5G, and 6G. In future work, we are interested to investigate the randomly modulated almost periodic arrays (Also in the presence of strong coupling).

\section{References}

1. Appendix B: Almost-periodic functions and their spectra . (2009). Available online: https://rd.springer.com/content/pdf/bbm:9781-84882-181-1\%2F1.pdf

2. Naum K. Berger, Spectral measurements with superresolution based on periodic modulation of the spectrum, APPLIED OPTICS, Vol. 47, No. 35 , 10 December 2008.

3. Naum K. Berger, Periodic modulation-based spectral and temporal superresolution with a single measurement, APPLIED OPTICS , Vol. 54, No. 10 , 1 April 2015

4. W. Janos, "Optimal filtering of periodic pulse-modulated time series," in IRE Transactions on Information Theory, vol. 5, no. 2, pp. 67-74, June 1959, doi: 10.1109/TIT.1959.1057494.

5. A. Pollastri, Periodic Structures EE625 Periodic Structures and Floquet's Theorem Periodic Structures .

6. K. Watanabe, Study on Spectral-Domain Formulation of Electromagnetic Scattering by Periodic Strip Array with Period Modulation, 2019 IEEE Conference on Antenna Measurements Applications (CAMA), 2019, pp. 165-166, doi: 10.1109/CAMA47423.2019.8959657.

7. B. Hamdi; T. Aguili, Spectral Floquet analysis devoted to meta-surface applied for $5 \mathrm{G}$ and planned 6G antenna designs, 2021 IEEE 19th International Symposium on Antenna Technology and Applied Electromagnetics (ANTEM), 2021, pp. 1-2, doi: 10.1109/ANTEM51107.2021.9518709.

8. B. Hamdi; T. Aguili, MoM-GEC combined with Floquet analysis to study scanned coupled almost periodic antenna arrays in massive MIMO for 5G generation and FMCW automotive radar applications, 2020 IEEE International Symposium on Antennas and Propagation and North American Radio Science Meeting, 2020, pp. 1941-1942, doi: 10.1109/IEEECONF35879.2020.9330171.

9. B.Hamdi ; T. Aguili and Junwu.Tao, Modélisation des circuits presque-périodiques , Éditions universitaires européennes (2 avril 2019) , pp.156 pages, ISBN-10: 6138476387- ISBN-13: 978-6138476382.

10. B. Hamdi; T. Aguili ; H. Baudrand, Floquet Modal Analysis To Modelize and Study 2-D Planar Almost Periodic Structures In Finite And Infinite Extent With Coupled Motifs, Progress In Electromagnetics Research B, vol. 62, pp. 63-86, 2015.

11. Mekkioui; Z. ;H. Baudrand, Bi-periodic centered-fed microstrip leaky-wave antenna (LWA) analysis by a source modal decomposition in spectral domain, IET, 1141-1149, 2009.

12. Baudrand, H.; M. Titaouine; N. Raveu; G. Fontgland, Electromagnetic modeling of planar almost periodic structures, SBMOI/IEEE MTT-S International Microwave and Optoelectronics Conference, 427-431, 2009.

13. Azizi, M. K.; L. Latrach; N. Raveu; A. Gharsallah; H. Baudrand, A new approach of almost periodic lumped elements circuits by an iterative method using auxiliary sources, American Journal of Applied Sciences, Vol. 10, No. 11, 1457-1472, 2013

14. Hamdi, B.; T. Aguili; N. Raveu; H. Baudrand, Calculation of the mutual coupling parameters and their effects in 1-D planar almost periodic structures, Progress In Electromagnetics Research B, Vol. 59, 269-289, 2014.

15. N.B. Latifa; T. Aguili, Synthesis and Optimization of Almost Periodic Antennas Using Floquet Modal Analysis and MoM-GEC Method, Journal of Electromagnetic Analysis and Applications, 2019, 11, 1-16

16. Abdallah, Y.; C. Menudier; M. Thevenot; T. Monediere, Investigations of the effects of mutualcoupling in reflectarray antennas,IEEE Antennas and Propagation Magazine, Vol. 55, No. 2, April2013.

17. Ishimaru, A.; R. J. Coe; G. E. Miller; W. P. Green, Finite periodic structure approach to large scanning array problems, IEEE Trans. Antennas Propagat., 1985.

18. Valerio, G.; P. Baccarelli; P. Burghignoli; A. Galli; R. Rodrguez-Berral; F. Mesa, Analysis of periodic shielded microstrip lines excited by nonperiodic sources through the array scanning method,Radio Science, Vol. 43, 2008.

19. Rodrguez-Berral; R., F. Mesa; P. Baccarelli; P. Burghignoli, Excitation of a periodic microstrip line by an aperiodic delta-gap source, IEEE Trans. Antennas Propagat. Letters, Vol. 8, 2009.

20. X. Dardenne ; C. Craeye, Application of the Array Scanning Method with Windowing to the Analysis of Finite Rectangular Periodic Structures," 2005 18th International Conference on Applied Electromagnetics and Communications, 2005, pp. 1-4, doi: 10.1109/ICECOM.2005.205022.

21. C. Craeye ; D. González-Ovejero, A review on array mutual coupling analysis, Radio science , VOL. 46, RS2012, doi:10.1029/2010RS004518, 2011.

22. Craeye, C., Expoitation of infinte-array results for accurate solution of finite widebands arrays, EMTS, 2007.

23. F. Mesa; C. di Nallo ; D. R. Jackson, The theory of surface-wave and space-wave leaky-mode excitation on microstrip lines, in IEEE Transactions on Microwave Theory and Techniques, vol. 47, no. 2, pp. 207-215, Feb. 1999, doi: 10.1109/22.744296.

24. Capolino, F. (2009), Theory and Phenomena of Metamaterials, (1st ed.). CRC Press. https://doi.org/10.1201/9781420054262

25. Skrivervik, K. ; L. R. Mosig, Finite phased array of microstrip patch antennas: The infinite array approach, IEEE Trans. Antennas Propagat., 1992. 


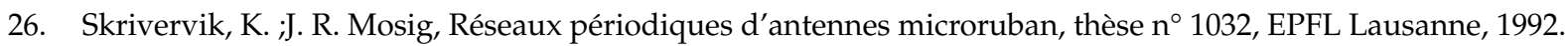

27. Sze, K. Y. ; L. Shafai, Reflection properties of infinite periodic arrays of rectangular conducting patches, Can. J. Elect. Comput. Eng., Vol. 24, No. 1, Jan. 1999.

28. Vardaxoglou, J. C., Frequency Selective Surfaces, Analysis and Design, John Wiley and Sons, 1997.

29. Mekkioui, Z. ; H. Baudrand, A full-wave analysis of uniform microstrip leaky-wave antenna with arbitrary metallic strips, Electromagnetics, Vol. 28, No. 4, 296-314, 2008.

30. Watanabe, K., Spectral-domain approach to electromagnetic scattering from imperfectly periodic, 13th International Conference on Mathematical Methods in Electromagnetic Theory, 1-6, Ukraine, Sep. 2010.

31. Watanabe, K. ; K. Yasumoto, Two-dimensional electromagnetic scattering of non-plane incident waves by periodic structures, Progress In Electromagnetics Research, Vol. 74, 241-271, 2007.

32. Bhattacharyya, K. A., Phased Array Antennas: Floquet Analysis, Synthesis, BFNs, and Active Array Systems, Wiley and Sons, Mar. 2006.

33. De Sabata, A.; Matekovits, L. ; Lipan, O. (2017), Band pattern of commensurate modulated periodic structures, IET Microw. Antennas Propag., 11: 1303-1307.

34. Salazar-Arrieta, J.D.; Halevi, P. (2021), Wave Propagation in Electric Periodic Structure in Space with Modulation in Time (2D+1). I. Theory.

35. A. M. Patel ;A. Grbic, A Printed Leaky-Wave Antenna Based on a Sinusoidally-Modulated Reactance Surface. in IEEE Transactions on Antennas and Propagation, vol. 59, no. 6, pp. 2087-2096, June 2011, doi: 10.1109/TAP.2011.2143668.

36. A. Oliner ; A. Hessel, Guided waves on sinusoidally-modulated reactance surfaces. in IRE Transactions on Antennas and Propagation, vol. 7, no. 5, pp. 201-208, December 1959, doi: 10.1109/TAP.1959.1144771.

37. Patch Antenna Array for FMCW Radar - MATLAB and Simulink, 2019.

Available online: https://fr.mathworks.com/help/phased/ug/patch-antenna-array-for-fmcw-radar.html

38. R. Kulke et al., 24 GHz Radar Sensor Integrates Patch Antennas, EMPC, 2005.

39. Ali Grami , Introduction to Digital Communications, Academic Press is an imprint of Elsevier, ISBN 978-0-12-407682-2 // doi10.1016/b978-0-12-407682-2.09985-3

40. Enrique A. González-Velasco, in Fourier Analysis and Boundary Value Problems, 1995

41. JULIUS O. SMITH III , Mathematics of the Discrete Fourier Transform (DFT), with Audio Applications - Second Edition, W3K

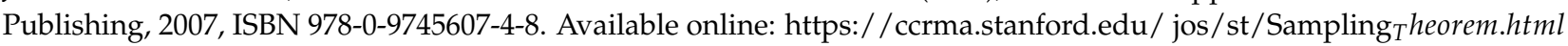

42. Prof. John A. Peacock, School of Physics and Astronomy-Fourier Analysis, Available online:

https://www.roe.ac.uk/japwww/teaching/fourier/fourier_lectures_part1.pdf 